\title{
Subunit Composition of Plasma von Willebrand Factor \\ Cleavage Is Present in Normal Individuals, Increased in IIA and IIB von Willebrand Disease, but Minimal in Variants with Aberrant Structure of Individual Oligomers (Types IIC, IID, and IIE)
}

Theodore S. Zimmerman, Judith A. Dent, Zaverio M. Ruggeri, and Louis H. Nannini

Department of Basic and Clinical Research, Scripps Clinic and Research Foundation, La Jolla, California 92037; Department of

Pathology, Methodist Hospital, Lubbock, Texas 79408

\begin{abstract}
We have evaluated the subunit composition of plasma von Willebrand factor (vWF) and found evidence that cleavage is present in normal individuals, increased in IIA and IIB von Willebrand disease (vWD), but decreased or absent in variants with aberrant structure of individual oligomers. vWF was rapidly purified from plasma on an analytical scale by monoclonal antibody immunoaffinity chromatography in the presence of protease inhibitors. After reduction and electrophoresis in 5\% polyacrylamide gels containing sodium dodecyl sulfate, fragments of 189,176 , and $140 \mathrm{kD}$, as well as the predominant $225-\mathrm{kD}$ subunit, were identified in plasma vWF from 25 normal individuals. The vWF polypeptides were detected by immunoblotting with a mixture of 55 anti-vWF monoclonal antibodies followed by ${ }^{125}$ I-rabbit anti-mouse antibody and autoradiography. In five individuals with type IIA and five individuals with type IIB vWD, the proportions of 176 and $140-\mathrm{kD}$ fragments were increased relative to the intact 225-kD subunit, as determined by excising each band and quantitating incorporated radioactivity. In contrast, these fragments were either not detectable or were present in only trace amounts in variants with abnormal structure of individual oligomers (types IIC and IID, and a new variant, type IIE vWD). The results reported here provide evidence that absence of large $\mathrm{VWF}$ multimers in these two groups of variants results from different mechanisms. In addition, they demonstrate that partial cleavage of the plasma vWF subunit is a normal event.
\end{abstract}

\section{Introduction}

Recently, several von Willebrand disease (vWD) ${ }^{1}$ variants have been identified in which large multimers are absent from plasma. These can be divided into two groups based on the structure of the individual oligomers constituting von Willebrand factor (vWF). In types IIA and IIB, the same bands are present as in normal plasma vWF although the fastest moving band in each oligomer is relatively increased, especially in type IIA (1). On

This work was originally published in abstract form, 1985. Blood. 66: 346a. This is publication no. 4059 BCR from the Research Institute of Scripps Clinic.

Address reprint requests to Dr. Zimmerman, Scripps Clinic and Research Foundation, 10666 North Torrey Pines Rd., La Jolla, CA 92037. Received for publication 26 August 1985.

1. Abbreviations used in this paper: PAGE, polyacrylamide gel electrophoresis; PMSF, phenylmethylsulfonyl fluoride; vWD, von Willebrand disease; vWF, von Willebrand factor.

J. Clin. Invest.

(c) The American Society for Clinical Investigation, Inc.

0021-9738/86/03/0947/05 \$1.00

Volume 77, March 1986, 947-951 the other hand, some normal bands are absent and/or new bands are present in types IIC (2-4), IID (5), and the new variant described in this report (type IIE).

Gralnick and co-workers (6) have suggested that proteolysis may play a role in the pathogenesis of type IIA vWD by showing that in vitro loss of multimers of intermediate size could be prevented by collecting blood from three IIA patients in the presence of leupeptin, $\mathrm{N}$-ethylmaleimide, and EDTA. These substances are effective in blocking the activity of the platelet calcium-activated protease, an enzyme which has been shown to cause loss of large multimers from normal vWF (7).

In order to evaluate the possible role of in vivo proteolysis in the pathogenesis of vWD variants, we rapidly purified vWF from plasma on an analytical scale in the presence of the abovementioned and other protease inhibitors. We then examined the subunit composition of plasma vWF by sodium dodecyl sulfatepolyacrylamide electrophoresis (SDS-PAGE) and anti-vWF monoclonal antibody immunoblotting. Our studies provide evidence that in vivo proteolytic cleavage of the vWF subunit is a normal occurrence. Although only a small proportion of plasma vWF was cleaved, this proportion was remarkably similar in different normal individuals. Plasma vWF cleavage was significantly increased over normal in each IIA and IIB vWD patient tested. These results are consistent with a role for proteolytic cleavage in causing the absence of large vWF multimers in IIA and IIB vWD. Moreover, since the relative proportion of the fastest band in each repeating oligomer is increased in these variants, it is likely that proteolysis contributes to this component of the pattern. On the other hand, little or no proteolysis was evident in vWD variants who lacked large multimers but in whom the structure of individual oligomers (types IIC and IID vWD) was aberrant. This was also true for a new variant described in the present report (type IIE vWD). Thus, a different mechanism must be responsible for the absence of large multimers in these individuals, as well as for their aberrant multimeric pattern.

\section{Methods}

Reagents. Chloromethyl ketone specific protease inhibitors, D-Phe-Lpro-L-Arg- $\mathrm{CH}_{2} \mathrm{Cl}$, dansyl-L-Glu-Gly-L-Arg- $\mathrm{CH}_{2} \mathrm{Cl}$, and D-Phe-L-Phe-LArg- $\mathrm{CH}_{2} \mathrm{Cl}$, were acquired from Behring Diagnostics, La Jolla, CA; leupeptin from Chemicon, El Segundo, CA; phenylmethylsulfonyl fluoride (PMSF), aprotinin, $N$-ethylmaleimide (NEM), and benzamidine from Sigma Chemical Co., St. Louis, MO; electrophoresis pure reagents were from Bio-Rad Laboratories, Richmond, CA; rabbit anti-mouse IgG from Zymed, South San Francisco, CA. All other reagents were of the highest grade available.

Preparation of plasma samples. Blood was drawn from an antecubital vein into polypropylene syringes and immediately transferred into polypropylene tubes containing one-tenth final volume of $3.8 \%$ sodium citrate, pH 7.4, with protease inhibitors. Three combinations of inhibitors were used: (a) leupeptin, $0.425 \mathrm{mM}$, and the three chloromethyl ketone in- 
hibitors, $0.01 \mathrm{mM}$ each; (b) leupeptin, $1.0 \mathrm{mM}$; EDTA, $5.0 \mathrm{mM}$; and $N$-ethylmaleimide, $6 \mathrm{mM}$; or (c) PMSF, $1 \mathrm{mM}$, benzamidine, $20 \mathrm{mM}$, and aprotinin, $20 \mathrm{U} / \mathrm{ml}$, used in conjunction with the leupeptin, EDTA, and $N$-ethylmaleimide.

Platelet-poor plasma was obtained by centrifugation of citrated blood at $3,000 \mathrm{~g}$ for $20 \mathrm{~min}$ at $23^{\circ} \mathrm{C}$. The plasma was rapidly separated from the cells and used either fresh or stored immediately at $-70^{\circ} \mathrm{C}$ until tested.

Monoclonal antibodies. The monoclonal antibodies used in this study were prepared as described previously (8) using the following immunogens: native vWF, reduced and alkylated intact $\mathrm{vWF}$, a reduced and alkylated 52-kD tryptic fragment of vWF (9), and a whole tryptic digest of vWF (9). All monoclonal antibodies used for immunoblotting were reactive with the intact $225-\mathrm{kD}$ subunit. To maximize reactivity, a pool of 55 anti-vWF monoclonal antibodies was prepared as the probe for these experiments. The antibody used for immunoisolation of vWF (5.5.72) was raised against native vWF and did not react with reduced vWF.

Immunoisolation of plasma $v W F$. Plasma samples were immunoisolated with anti-vWF monoclonal antibody 5.5.72 which had been coupled to cyanogen bromide-activated Sepharose CL4B beads (Pharmacia Fine Chemicals, Piscataway, NJ) at a ratio of $4 \mathrm{mg}$ of antibody/ $\mathrm{ml}$ of beads. Approximately $15 \mu \mathrm{g}$ of $\mathrm{vWF}$ bound/ $\mathrm{ml}$ of beads used.

Two variations of the immunoisolation technique were followed. The first was a batch method which was used to obtain sufficient vWF for 10-12 lanes of SDS-PAGE. It utilized $2.5 \mathrm{ml}$ of beads and $5 \mathrm{ml}$ of normal plasma and required a period of $5 \mathrm{~h}$ for completion. Plasma volumes were increased up to $10 \mathrm{ml}$ to compensate for the lower vWF antigen levels present in some patients. The beads were prewashed with $0.1 \mathrm{M}$ Tris-HCl, $0.15 \mathrm{M} \mathrm{NaCl}, 0.1 \%$ SDS pH 7.3, and inhibitors: $1 \mathrm{mM}$ leupeptin, $5 \mathrm{mM}$ EDTA, and $6 \mathrm{mM} N$-ethylmaleimide. These inhibitors were used in all subsequent washing and elution steps. After incubation of beads and plasma at $4^{\circ} \mathrm{C}$ for $2 \mathrm{~h}$ on a rotator, the beads were transferred to polypropylene columns (Bio-Rad Laboratories), washed first with 10 bed volumes of $0.5 \mathrm{M} \mathrm{LiCl}, 0.1 \mathrm{M}$ Tris-HCl ( $\mathrm{pH} \mathrm{8.0)}$ (buffer A), and then, with 10 volumes of $0.02 \mathrm{M} \mathrm{NaCl}, 0.05 \mathrm{M}$ Tris- $\mathrm{HCl}$ (pH 8.0) (buffer B). Following removal of the beads from the columns, the vWF was eluted by incubation with $2 \mathrm{ml}$ of $2 \%$ SDS, $0.1 \mathrm{M}$ Tris, pH 8.0, at $60^{\circ} \mathrm{C}$ for $20 \mathrm{~min}$. Beads were removed by centrifugation at $7,000 \mathrm{~g}$ for $2 \mathrm{~min}$ and filtration of the eluate through a $0.2-\mu \mathrm{m}$ filter (Gelman-Acrodisc, Ann Arbor, MI). Using Amicon Centricon 30 microconcentrators (Amicon Corp., Danvers, MA), the eluate volumes were reduced to $\sim 100 \mu \mathrm{l}$ by centrifugation at $5,000 \mathrm{~g}$ for $2 \mathrm{~h}$ at $20^{\circ} \mathrm{C}$. Between 5 and $10 \mu \mathrm{l}$ were subsequently applied to each SDS-PAGE lane after reduction as described below.

The second technique was performed more rapidly but provided vWF sufficient only for 1 to 2 SDS-PAGE lanes. $250 \mu \mathrm{l}$ of beads and 1$\mathrm{ml}$ volumes of normal plasma were used, allowing analysis of samples on SDS-PAGE within $2 \mathrm{~h}$ of drawing blood. Plasma was passed over the beads in a mini-column (VWR Scientific, subsidiary of Univar, Los Angeles, CA). The beads were then washed with 10 bed volumes of buffer $A$ and 10 bed volumes of buffer B (see above). Following this, $150 \mu \mathrm{l}$ of elution buffer was added, and the column was capped at both ends and heated at $60^{\circ} \mathrm{C}$ for $20 \mathrm{~min}$. The beads were then removed from the column, and the supernatant $(\sim 150 \mu \mathrm{l})$ was obtained by centrifugation at $7,000 \mathrm{~g}$ for $2 \mathrm{~min}$. Depending on the vWF concentration, either 75 or $150 \mu \mathrm{l}$ were then applied to each SDS-PAGE lane after reduction as described below. The multimeric patterns obtained with either technique were identical.

SDS-PAGE and immunoblotting. 5\% SDS-polyacrylamide gels were prepared as described by Laemmli (10). vWF was reduced in a final concentration of $65 \mathrm{mM}$ dithiothreitol for $15 \mathrm{~min}$ at $60^{\circ} \mathrm{C}$. The molecular mass markers used to calculate the apparent molecular masses of the subunits in this system were fibronectin, $220 \mathrm{kD}$; myosin, $200 \mathrm{kD}$; beta galactosidase, $116 \mathrm{kD}$; phosphorylase $\mathrm{B}, 92.5 \mathrm{kD}$; and bovine serum albumin, $66.2 \mathrm{kD}$.

Transfer of proteins from the polyacrylamide gel onto nitrocellulose membrane was performed using a $25 \mathrm{mM}$ Tris, $192 \mathrm{mM}$ glycine, $20 \%$ (vol/vol) methanol buffer, at $0.25 \mathrm{amp}, 3^{\circ} \mathrm{C}$ for $16 \mathrm{~h}$. Nitrocellulose membranes were labelled using a sandwich of the anti-vWF monoclonal antibody pool, followed by ${ }^{125}$ I-rabbit anti-mouse IgG according to the method of Johnson and co-workers (11). Bands were visualized by autoradiography with Kodak XRP-1 film (Eastman Kodak Co., Rochester, $\mathrm{NY}$ ) and quantified after excision, using a Packard auto-gamma scintillation spectrometer (Packard Instrument Co., Downers Grove, IL).

SDS-agarose electrophoresis. SDS-agarose electrophoresis was performed as described previously (1) using $1.4 \%$ low gelling temperature agarose cast in 9-cm gels.

\section{Results}

Following reduction, SDS-PAGE and immunoblotting with the pool of monoclonal antibodies, vWF fragments with mobility corresponding to 189,176 , and $140 \mathrm{kD}$ were consistently detected in normal plasma, in addition to the predominant intact 225-kD subunit (Figs. 1 and 2). The relative proportions of these four species were quite similar in each of the 25 normal individuals tested (Fig. 3). All of these plasmas were from blood collected and subsequently processed in the presence of the leupeptin, $N$-ethylmaleimide, and EDTA combination.

However, similar results were also seen when the other inhibitor combinations were used (i.e., chloromethyl ketones and leupeptin, sometimes in conjunction with benzamidine, PMSF, and aprotinin). Normal plasma collected in the absence of inhibitors also gave similar results. Nevertheless, patient samples were always collected in the presence of inhibitors. Fresh normal plasma and frozen plasma which had been thawed once or twice had identical proportions of the $225-\mathrm{kD}$ subunit and smaller fragments. However, repeated freeze-thawing increased the proportion of fragments. Therefore, only fresh plasma, or frozen plasma which had not been thawed previously, was used for these studies.

Identification of vWF polypeptides by immunoblotting was compared with Coomassie blue staining of polyacrylamide gel lanes parallel to those which were immunoblotted (Fig. 1). For this purpose, vWF purified by agarose gel filtration of pooled cryoprecipitate (12), as well as vWF immunopurified from a single normal plasma, was used. Immunoblotting showed that both vWF preparations contained the 225, 189-, 176-, and 140$\mathrm{kD}$ species in similar proportions. Direct staining of the polyacrylamide gels demonstrated faintly staining bands at the 176and $140-\mathrm{kD}$ position. The $189-\mathrm{kD}$ polypeptide could not be visualized by Coomassie blue. Several contaminant polypeptides, not reacting with the anti-vWF monoclonal antibodies, were visible in the immunopurified vWF preparation (Fig. 1).

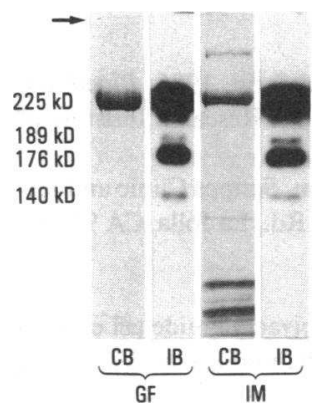

Figure 1. vWF polypeptides identified by immunoblotting or by direct staining of polyacrylamide gels with Coomassie blue. $\mathrm{vWF}$ isolated by agarose gel filtration of pooled cryoprecipitates (GF) (left) was compared with that obtained from $5 \mathrm{ml}$ of an individual normal plasma by immunoisolation (IM) (right) after reduction and SDS-PAGE. Approximately $20 \mu \mathrm{g}$ of protein were applied to each lane for Coomassie blue staining and $\sim 2 \mu \mathrm{g}$ for immunoblotting. Parallel polyacrylamide gel lanes were cut out for immunoblotting (IB) or for direct Coomassie blue staining (CB). The apparent molecular mass of each polypeptide is indicated on the left. The direction of electrophoresis is from top to bottom. The arrow in this and succeeding figures indicates the interface between the stacking gel (above) and running gel (below). 


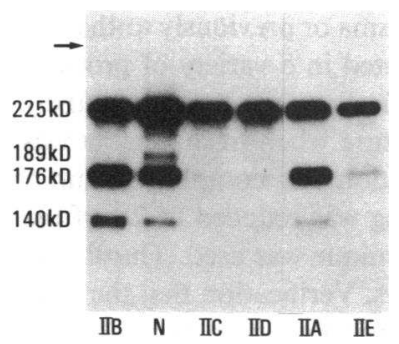

Additional evidence that all of the anti-vWF reactive polypeptides in the immunoisolated preparations were vWF-derived was obtained by subjecting plasma from a patient with severe vWD to the immunoisolation procedure in parallel with two normal plasmas. None of the anti-vWF immunoreactive polypeptides present in the normal plasma eluates could be detected in the severe vWD eluate (Fig. 4).

In order to provide evidence that proteolysis was not occurring during the immunoisolation process, multimeric analysis was performed on the fresh normal plasma samples and compared with the resultant affinity-purified vWF (Fig. 5). Although there was a slight decrease in the proportion of large multimers, all were still present after purification. More importantly, there was no alteration in the relative intensity of the bands in each repeating oligomer. Each band was present in the same proportion as seen in the starting plasma, and there was no shift toward the pattern seen in IIA vWD (i.e., increased intensity of the fastest moving band). In addition, affinity purification of patient vWF did not change the proportion of intermediate to small multimers, nor did it change the proportions of any of the bands composing individual oligomers (Fig. 5).

$v W F$ fragments in previously described $v W D$ variants. The vWF subunit composition of five individuals with IIA vWD, five individuals with IIB vWD, one with IIC vWD, and four

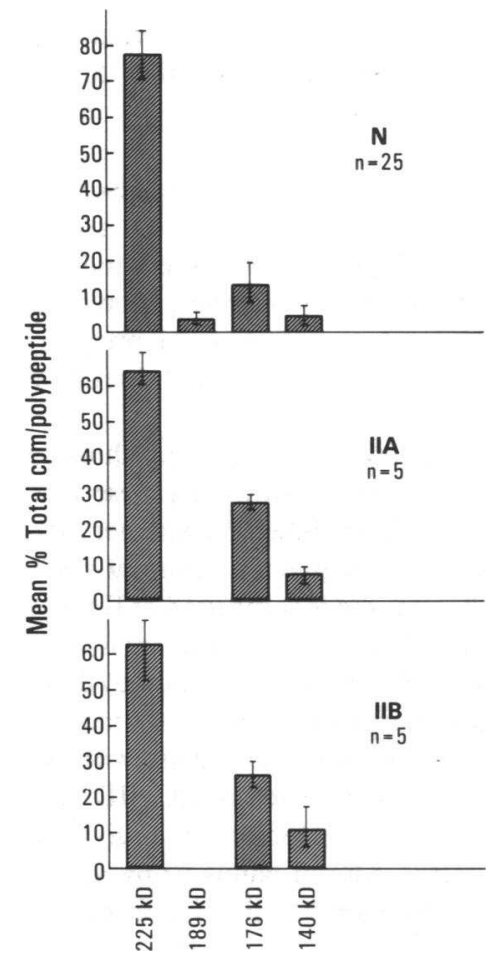

Figure 3. Relative concentration of $\mathrm{VWF}$ polypeptides of normal vWF as compared with vWF from IIA and IIB vWD as determined by excision of immunoblot bands and measuring incorporated radioactivity in a gamma counter. The ranges are shown by the brackets.

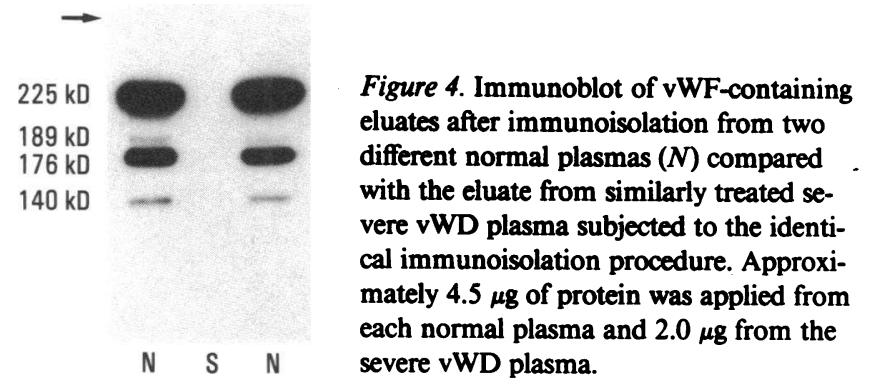

with IID vWD was compared with that of normal vWF. The most striking finding was the absence, or presence in only trace amounts, of any of the vWF fragments in IIC and IID vWD (Fig. 2). Both of these variants have aberrant structure of individual vWF oligomers (Fig. 6). This contrasted with IIA and IIB vWD in which the structure of individual oligomers was similar to normal with the exception that there was an increase in the relative proportion of the fastest migrating member of each oligomer. In this group, the proportion of the 176-kD fragment was increased in all samples and that of the $140-\mathrm{kD}$ fragment in most. Although these conclusions were evident on simple inspection of the autoradiograph, they were quantitatively verified by counting the radioactivity of each band after cutting it out of the nitrocellulose (Fig. 3). When each group of patients was compared with the group of normals, the increased proportion of both fragments was highly significant for both IIA and IIB vWD $(P<0.001$ for the 176 - and $140-\mathrm{kD}$ fragments by Student's $t$ test). Prolonged exposure of autoradiographs was necessary to visually demonstrate minor bands (i.e., those of 189 and $140 \mathrm{kD}$ ). This gave a falsely enhanced impression of the proportion of fragments compared with the $225-\mathrm{kD}$ subunit. Even with prolonged exposure, the $189-\mathrm{kD}$ fragment was not identified in IIA nor IIB vWF.

Type IIE: a new variant of $v W D$ with an aberrant multimeric pattern and reduced cleavage of plasma $v W F$. A new variant with aberrant structure of individual oligomers, designated type IIE, was also studied by this technique. Like the other variants with aberrant structure of individual oligomers, the type IIE also fell into the reduced cleavage category (Fig. 2). The propositus

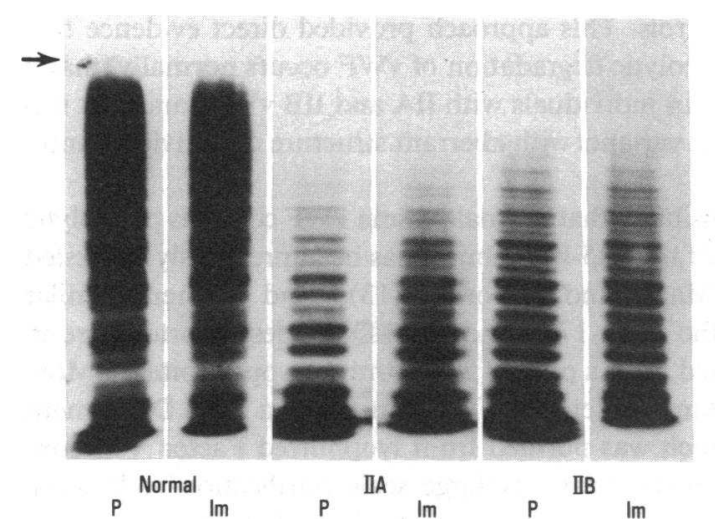

Figure 5. SDS-agarose multimeric analysis of vWF in plasma $(P)$ and after immunopurification (IM). The results from normal plasma, IIA plasma, and IIB plasma are shown. The direction of electrophoresis is from top to bottom. 


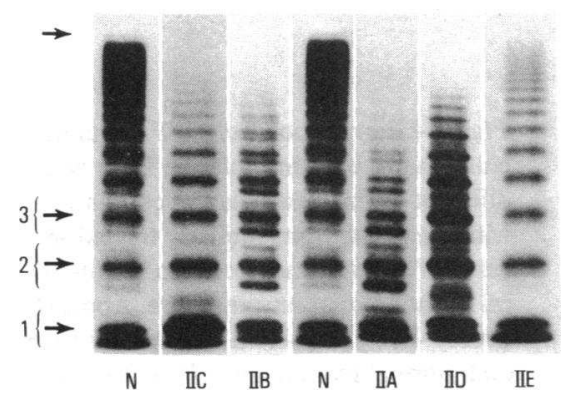

Figure 6. SDS-agarose multimeric analysis of plasma vWF from a normal individual compared with vWF of the vWD variants studied here. The three smallest individual oligomers are indicated by the brackets. The arrows point to the predominant band in each normal oligomer. In IIA and IIB vWD, the fastest moving band in each oligomer is increased relative to the predominant band. However, bands with similar mobilities to all normal bands are present. In types IIC, IID and IIE, either some normal bands are missing or there are bands present which are not seen in normal plasma.

was a 12-yr-old boy with a mild bleeding diathesis consistent with vWD. The bleeding time was $>20 \mathrm{~min}$ (normal, $<8.5 \mathrm{~min}$ ); the vWF:antigen, $0.26 \mathrm{U} / \mathrm{ml}$ (normal, $>0.45 \mathrm{U} / \mathrm{ml}$ ); the ristocetin cofactor, $0.30 \mathrm{U} / \mathrm{ml}$ (normal, $>0.45 \mathrm{U} / \mathrm{ml}$ ); and the Factor VIII procoagulant activity, $1.00 \mathrm{U} / \mathrm{ml}$ (normal, $>0.5 \mathrm{U} / \mathrm{ml}$ ). The large oligomers were missing from plasma (Fig. 6). The pattern of individual multimers was aberrant in that there was only one clearly identifiable band, rather than the repeating series of bands seen in normal vWF. The pattern differed from IIC vWD in that there was no intervening band. In addition, a marked increase in the smallest oligomer, a hallmark of type IIC, was not present.

The propositus' mother, though asymptomatic, showed similar abnormalities of vWF. Her vWF:antigen was $0.22 \mathrm{U} /$ $\mathrm{ml}$; ristocetin cofactor, $0.11 \mathrm{U} / \mathrm{ml}$; and Factor VIII procoagulant activity, $1.00 \mathrm{U} / \mathrm{ml}$. She also lacked large multimers in plasma and had an aberrant pattern of individual oligomers identical to that of her son.

\section{Discussion}

The technique reported here allows assessment of the subunit composition of vWF with a minimum of manipulation and from amounts of plasma that are readily obtained from patients and normal controls. This approach provided direct evidence that in vivo proteolytic degradation of vWF occurs normally, that it is increased in individuals with IIA and IIB vWD, and that it is decreased in variants with aberrant structure of individual multimers.

The possibility that normal plasma vWF contains proteolytic fragments of the $225-\mathrm{kD}$ subunit has been previously suggested by others. Martin and co-workers (13) found fragments similar in size to the 189-, 176-, and 140-kD species reported here in vWF purified from a pool of 200 normal cryoprecipitates. More recently, Hamilton et al. (14) demonstrated a 168-kD fragment in vWF'which was purified from lyophilized Factor VIII concentrates. In the relatively large scale purification techniques used by these investigators, the possibility of in vitro proteolysis could not be readily addressed. The analytical scale vWF isolation reported here allowed inclusion of a number of precautions to minimize in vitro proteolysis as a cause for fragmentation. First, isolations were either from fresh plasma or previously unthawed frozen plasma that had been collected in a variety of protease inhibitors. Secondly, the entire purification process was routinely completed within $6 \mathrm{~h}$ of venepuncture when fresh plasma was used. With frozen plasma, purification was complete within 5 $\mathrm{h}$ of thawing the plasma. Processing was reduced to $2 \mathrm{~h}$ if the smaller scale immunoisolation technique was used. Thirdly, all buffers contained protease inhibitors. Verification that the fragments were vWF derived was accomplished here by their identification in immunoblots with the pool of anti-vWF monoclonal antibodies. The use of monoclonal antibodies obviated the possibility that antibodies to contaminants in the original immunogen were reacting with irrelevant polypeptides in the immunoblots. This possibility was further excluded by the absence of any immunoreactive peptides in the eluates obtained when severe vWD plasma was subjected to the immunoisolation procedure (Fig. 4).

Gralnick and co-workers (6) inferred that the vWF of three IIA patients was more susceptible to in vitro proteolysis than normal vWF by showing that loss of intermediate multimers could be prevented by including leupeptin, $N$-ethylmaleimide, and EDTA in the anticoagulant into which blood was drawn. In the IIA patients studied here, inclusion of these inhibitors had little or no discernible effect on the multimeric structure of plasma vWF (Figs. 5 and 6). Nevertheless, our evidence that in vivo proteolysis of IIA vWF is greater than that of normal vWF supports the concept that IIA vWF has a heightened susceptibility to proteolytic attack.

However, the presence of increased vWF proteolysis in IIB vWD was not expected. IIB vWF clearly differs from IIA vWF in that it has an increased affinity for a platelet membrane receptor as compared with normal vWF (15), whereas IIA vWF reacts less well with platelets than normal vWF (16-17). In fact, direct interaction of type IIB vWF with platelets has been demonstrated $(15,18-19)$. It is possible that the increased fragmentation of IIB vWF is not the result of increased susceptibility to proteolytic attack, but rather results from its interaction with platelets and consequent cleavage by the platelet calcium-activated protease or other enzymes. The absence of the $189-\mathrm{kD}$ fragment from both IIA and IIB plasma vWF suggests that it had been further proteolyzed in vivo.

IIE vWD is clearly distinguished from previously described variants. Whereas some IIC families show only one band in each oligomer $(3,4)$, as does the IIE, all IIC patients have a marked increase in the fastest migrating (smallest) oligomer. Moreover, IIC families exhibit recessive inheritance, whereas the presently described IIE trait reported here displays autosomal dominant inheritance.

All of the vWD variants studied here share one thing in common, the absence of large multimers. However, these variants can now be divided into two groups based on whether their vWF is cleaved to a greater or lesser degree than normal vWF. The group with increased vWF cleavage is comprised of IIA and IIB vWD, in which the structure of individual oligomers is characterized by the same bands seen in normal vWF, but they differ only in that there is an increased proportion of the fastest moving band in each oligimer. On the other hand, the group with decreased or undetectable cleavage is comprised of IIC, IID, and IIE vWD, all of which have aberrant structure of individual oligomers with either some of the normal bands being absent or with bands present which have a mobility differing from nor- 
mal. These results suggest that the normal structure of individual vWF oligomers, as well as the exaggerated form seen in IIA and IIB, is, at least in part, the result of in vivo proteolysis. And while proteolysis may be responsible for the absence of large multimers in IIA and IIB vWD, one or more additional mechanisms must be postulated for their absence in IIC, IID, and IIE vWD.

\section{Acknowledgments}

We are grateful to Dr. Carol Kasper (University of Southern California, Los Angeles, CA) for provision of IIA plasma; Dr. Inga Marie Nilsson, (Department for Coagulation Disorders, Allmanna Sjukhuset, Malmo, Sweden) for IIC and IID plasma; Dr. Charles Abildgaard (University of California at Davis, CA) for IID plasma; Dr. Wahid Hanna (The University of Tennessee Memorial Research Center and Hospital, Knoxville, TN) for IID plasma; and Dr. H. Niessner (I. Medizinische Universsitatsklinik, A-1090 Wien, Lazarettgasse 14, West Germany) for IIB plasma. We, also, thank Ms. Claire Jackson and Ms. Ruth Stewart for the manuscript preparation.

This work was supported in part by grants HL15491 and HL31950 from the National Institutes of Health.

\section{References}

1. Ruggeri, Z. M., and T. S. Zimmerman. 1981. The complex multimeric composition of Factor VIII/von Willebrand factor. Blood. 57: $1140-1143$.

2. Ruggeri, Z. M., I. M. Nilsson, R. Lombardi, L. Holmberg, and T. S. Zimmerman. 1982. Aberrant multimeric structure of von Willebrand factor in a new variant of von Willebrand's disease (Type IIC). $J$. Clin. Invest. 70:1124-1127.

3. Mannucci, P. M., R. Lombardi, F. Pareti, S. Solinas, M. G. Mazzucconi, and G. Mariani. 1983. A variant of von Willebrand's disease characterized by recessive inheritance and missing triplet structure of von Willebrand factor multimers. Blood. 62:1000-1005.

4. Batlle, J., M. F. Lopez-Fernandez, J. Lasierra, A. Fernandez Villamor, C. Lopez Berges, A. Lopez Borrasca, Z. M. Ruggeri, and T. S. Zimmerman. 1986. von Willebrand's disease Type IIC with different abnormalities of von Willebrand factor in the same sibship. Am. J. Hematol. 21:177-188.

5. Kinoshita, S., J. Harrison, J. Lazerson, and C. F. Abildgaard. 1984. A new variant of dominant Type II von Willebrand's disease with aberrant multimeric pattern of Factor VIII-related antigen (Type IID). Blood. 63: 1369-1371.

6. Gralnick, H. R., S. B. Williams, L. P. McKeown, P. Maisonneuve, C. Jenneau, Y. Sultan, and M. E. Rick. 1985. In vitro correction of the abnormal multimeric structure of von Willebrand factor in type IIA von Willebrand's disease. Proc. Natl. Acad. Sci. USA. 82:5968-5972.

7. Kunicki, T. J., R. R. Montgomery, and J. Shulleck. 1985. Cleavage of human von Willebrand factor by platelet calcium-activated protease. Blood. 65:352-356.

8. Fulcher, C. A., and T. S. Zimmerman. 1982. Characterization of the human Factor VIII procoagulant protein with a heterologous precipitating antibody. Proc. Natl. Acad. Sci. USA. 79:1648-1652.

9. Fujimura, Y., K. Titani, L. Z. Holland, S. Russell, J. R. Roberts, J. H. Elder, Z. M. Ruggeri, and T. S. Zimmerman. 1986. Von Willebrand factor: a reduced and alkylated $52 / 48 \mathrm{kDa}$ tryptic fragment beginning at amino acid residue 449 contains the domain interacting with platelet glycoprotein Ib. J. Biol. Chem. 261:381-385.

10. Laemmli, U. K. 1970. Cleavage of structural proteins during the assembly of the head of bacteriophage $T_{4}$. Nature (Lond.). 227:680-685.

11. Johnson, D. A., J. W. Gautsch, J. R. Sportsman, and J. H. Elder. 1984. Improved technique utilizing nonfat dry milk for analysis of proteins and nucleic acids transferred to nitrocellulose. Gene. Anal. Techn. 1:3-8.

12. De Marco, L., and S. S. Shapiro. 1981. Properties of human asialo-Factor VIII. A ristocetin-independent platelet-aggregating agent. J. Clin. Invest. 68:321-328.

13. Martin, S. E., V. J. Marder, C. W. Francis, L. S. Loftus, and G. H. Barlow. 1980. Enzymatic degradation of the factor VIII-von Willebrand factor protein: a unique tryptic fragment with ristocetin cofactor activity. Blood. 55:848-858.

14. Hamilton, K. K., L. J. Fretto, D. S. Grierson, and P. A. McKee. 1985. Effects of plasmin on von Willebrand factor multimers. Degradation in vitro and stimulation of release in vivo. J. Clin. Invest. 76:261-270.

15. De Marco, L., A. Girolami, T. S. Zimmerman, and Z. M. Ruggeri. 1985. Interaction of purified IIB von Willebrand factor with the platelet membrane glycoprotein Ib induces fibrinogen binding to the glycoprotein IIb/IIIa complex and initiates aggregation. Proc. Natl. Acad. Sci. USA. 82:7424-7428.

16. Ruggeri, Z. M., F. I. Pareti, P. M. Mannucci, N. Ciavarella, and T. S. Zimmerman. 1980. Heightened interaction between platelets and Factor VIII/von Willebrand Factor in a new subtype of von Willebrand's disease. N. Engl. J. Med. 302:1047-1051.

17. Ruggeri, Z. M., and T. S. Zimmerman. 1980. Variant von Willebrand's disease. Characterization of two subtypes by analysis of multimeric composition of Factor VIII/von Willebrand factor in plasma and platelets. J. Clin. Invest. 65:1318-1325.

18. Holmberg, L., I. M. Nilsson, L. Borge, M. Gunnarson, and E. Sjorin. 1983. Platelet aggregation induced by 1-Desamino-8-D-arginine vasopressin (DDAVP) in Type IIB von Willebrand's disease. $N$. Engl. $J$. Med. 309:816-821.

19. Saba, H. I., S. R. Saba, J. Dent, Z. M. Ruggeri, and T. S. Zimmerman. 1985. Type IIB Tampa: a variant of von Willebrand disease with chronic thrombocytopenia, circulating platelet aggregates, and spontaneous platelet aggregation. Blood. 66:282-286. 